\title{
Design of Surveillance Sensor Grids with a Lifetime Constraint
}

\author{
Vivek Mhatre $^{1}$, Catherine Rosenberg ${ }^{1}$, Daniel Kofman ${ }^{2}$, Ravi Mazumdar ${ }^{1}$, and \\ Ness Shroff ${ }^{1}$ \\ 1 School of Electrical and Computer Engineering, Purdue University \\ West Lafayette, IN-47906, USA \\ \{mhatre, cath, mazum, shroff\}@ecn.purdue.edu \\ 2 ENST, Paris France \\ $\{$ daniel.kofman\}@enst.fr
}

\begin{abstract}
A surveillance area is to be monitored using a grid network of heterogeneous sensor nodes. There are two types of nodes; type 0 nodes which perform sensing and relaying of data within a cluster, and type 1 nodes which act as cluster heads or fusion points. A surveillance aircraft visits the area periodically, and gathers information about the activity in the area. During each data gathering cycle, the sensor nodes use multihopping to communicate with their respective cluster heads, while the cluster heads perform data fusion, and transmit the aggregated data directly to the aircraft. We formulate and solve a cost based optimization problem to determine the optimum number of sensor nodes $\left(n_{0}\right)$, cluster head nodes $\left(n_{1}\right)$ and the battery energy in each type of nodes ( $E_{0}$ and $E_{1}$ respectively) to ensure at least $T$ data gathering cycles. We observe that the number of cluster heads required, $n_{1}$, scales approximately as $n_{0}{ }^{1-\frac{k}{4}}$ where $k$ is the propagation loss exponent.
\end{abstract}

\section{Introduction}

The need for remote sensing of phenomena of interest has recently led to a surge of interest in the field of wireless sensor networks. Such networks are made up of inexpensive sensor nodes which use wireless channel to communicate with each other as well as with the base station. Sensor networks have useful applications in both military as well as civilian domains [1]. Civilian applications of sensor networks consist of smart homes, temperature control in buildings, seismic measurements, habitat monitoring etc. Military applications of sensor networks are battlefield assessment, surveillance of sensitive areas, intrusion detection etc. The application that we study in this paper is the surveillance of a sensitive area. In such applications sensor nodes are placed along the points of a grid over an area which is to be monitored. Each node has a sensing as well as communication radius of $r$. If the two radii are different, our analysis can be easily modified to take that into account. The nodes are organized as clusters with a single cluster head for each cluster. We call the ordinary sensor nodes to be of type 0 while the cluster head nodes to be of type 1 . The number of type 0 nodes used is $n_{0}$, 
and each has a battery energy of $E_{0}$, while the number of type 1 nodes used is $E_{1}$, and each has a battery energy of $E_{1}$. A surveillance aircraft (possibly unmanned) visits the area periodically to gather updates from the area. When the aircraft arrives, the sensor nodes send their information to their respective cluster heads using multi-hop communication. The cluster heads aggregate the received data, and send the aggregated data to the aircraft using a single hop transmission. Each such data gathering phase drains energy from the batteries of sensor nodes. Nodes are assumed to have a finite battery lifetime. For this setting, we would like to guarantee a certain minimum number of data gathering trips of the aircraft which we call the lifetime of the network. A sensor system becomes unusable when connectivity of nodes or sensing coverage of the area can no longer be ensured. We would like to minimize the cost of the entire network while ensuring node connectivity and sensing coverage of the region. We find that the required number of type 1 nodes $n_{1}$ scales approximately as $n_{0}{ }^{1-\frac{k}{4}}$ where $k$ is the propagation loss exponent.

This paper is organized as follows. In Section 2 we discuss some related work on similar problems. In Section 3 we formulate and solve our design problem. We present some numerical results of a case study in section 4 . Finally we conclude in Section 5.

\section{Related Work}

In [5] the authors study a scenario in which nodes are distributed randomly over a unit area. Nodes communicate with the cluster heads using a single hop transmission. The cluster heads aggregate the collected data and and send it to the base station using a single hop transmission. Cluster heads are rotated periodically for the purpose of load balancing. For this scenario, the authors minimize the total energy spent in the network to obtain an expression for the required cluster head density. However we note that for cluster head rotation to be possible, all the nodes should be identical, and should be capable of performing data fusion as well as transmissions over long distances. This leads to additional hardware complexity in all the nodes.

In [4], the authors study a random deployment of nodes where nodes use multi-hop communication to send their data to the cluster heads. The cluster heads aggregate the received data, and use multi-hop communication to send the aggregated data to the base station. For this setting, the authors minimize the total average energy spent in the network to determine the optimum cluster head intensity. However the authors do not provide any bounds on the lifetime of the network. In [2], the authors provide upper bounds on the lifetime of a sensor network. In [3], the authors present an optimum policy to assign nodes to the cluster heads in order to improve the network lifetime.

Our work is different from all the above studies because we study a scheme which uses heterogeneous sensor nodes (two types of nodes), and take into account the costs of the two types of nodes in the overall system design problem. 


\section{Problem Formulation}

We consider a surveillance sensor grid in which the network topology is very simple. We assume that it is possible to place the sensor nodes deterministically along grid points. While this may not always be possible, there are scenarios in military applications when we have complete control over node placement. We are interested in covering a region of unit area with sensor nodes. The nodes have a communication as well as sensing radius $r$, and are placed distance $r$ apart along the grid points. This ensures coverage of the area as well as node connectivity. We assume that $r<<1$, since in most practical applications we are interested in covering a given area with a large number of nodes; each of which has a sensing radius that is much smaller than the dimensions of the area. There are two types of nodes; type 0 nodes and type 1 nodes. Type 0 nodes are the sensor nodes, while type 1 nodes act as cluster heads besides acting as sensor nodes (see Figure 1). Let $n_{0}$ denote the number of sensor nodes and $n_{1}$ denote the number of cluster heads. A surveillance aircraft visits the area periodically

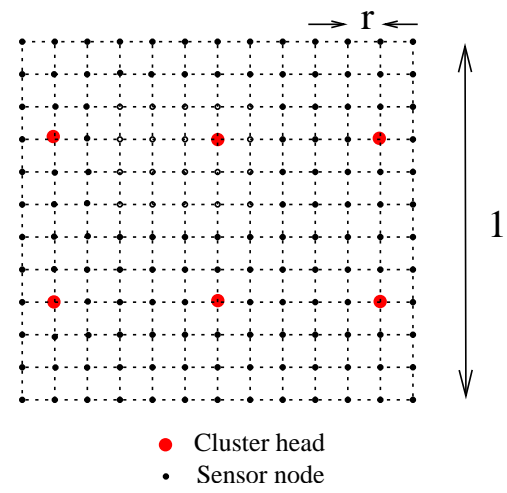

Fig. 1. A surveillance sensor grid.

and gathers information about the activity in the area. During each visit of the aircraft, each sensor node senses and sends a single data packet to its cluster head using multi-hop communication. The cluster head aggregates the received data packets into a single packet and transmits it to the aircraft. During each such data gathering phase, nodes spend a part of their battery energy for relaying of packets. Since the nodes have a finite battery energy there is an upper bound on the number of such data gathering cycles that can be sustained. Our aim is to guarantee a network lifetime of $T$, i.e., at least $T$ data gathering cycles while ensuring connectivity as well as coverage of the area.

We model the cost of each node as the sum of its hardware cost and its battery cost. Hence the cost of a type $i$ node $C_{i}$ is:

$$
C_{i}=\alpha_{i}+\beta E_{i}
$$


where $\alpha_{i}$ is the hardware cost of the node, $E_{i}$ is the battery energy of the node and $\beta$ is the proportionality constant for the battery cost. The reason we have different cost parameters $\alpha_{0}, \alpha_{1}$ and different battery energies $E_{0}, E_{1}$ is that the sensor nodes have a simpler functionality as compared to the cluster head nodes. The sensor nodes perform short range transmissions and do not have to perform any data aggregation. As against this, the cluster head nodes have to perform computations for data aggregation, and also perform long range transmissions to the aircraft. The cluster heads are also responsible for managing MAC and routing in the cluster. Hence the hardware cost, as well as the battery energy of the cluster head nodes are much higher than the sensor nodes.

There are $n_{0}$ type 0 nodes each with battery energy $E_{0}$ and $n_{1}$ type 1 nodes each with battery energy $E_{1}$. If we denote $\left[n_{0}, n_{1}, E_{0}, E_{1}\right]$ by $\bar{x}$, then the total cost of the network $f(\bar{x})$ is:

$$
\begin{aligned}
f(\bar{x}) & =n_{0}\left(\alpha_{0}+\beta E_{0}\right)+n_{1}\left(\alpha_{1}+\beta E_{1}\right) \\
& =n_{0} \alpha_{0}+n_{1} \alpha_{1}+\beta\left(n_{0} E_{0}+n_{1} E_{1}\right)
\end{aligned}
$$

Since nodes have a coverage/communication radius of $r$ and we are interested in covering a unit area with these nodes, the condition for coverage and connectivity is:

$$
n_{0}+n_{1} \geq \frac{1}{r^{2}}
$$

We observe that since the nodes use multi-hop communication to reach the cluster heads, the type 0 nodes which are one hop away from the cluster heads have the highest burden of relaying, since every packet sent to the base station has to go through them. We call these nodes the critical nodes. There are 4 critical nodes in each cluster (see Figure 2). ${ }^{3}$ We ignore the fact that the load on the critical nodes near the region boundary is likely to be less due to edge effects, and focus only on the clusters in the interior of the region.

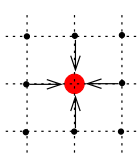

Fig. 2. Critical nodes

Since the critical nodes have the highest energy burden, these nodes are likely to drain their battery before other type 0 nodes. Besides the critical nodes, the

\footnotetext{
${ }^{3}$ If the sensing and communication radii are different, i.e., the communication radius (say $r_{c}$ ) is larger than the sensing radius (say $r_{s}$ ), the number of critical nodes is equal to the number of nodes that are within $r_{c}$ distance of the cluster head. For simplicity, we assume $r_{c}=r_{s}$ so that there are 4 critical nodes. However the case of $r_{c}>r_{s}$ can be handled similarly. Note that $r_{c}<r_{s}$ is not feasible since no communication is possible in that case.
} 
cluster heads also have high energy expenditure due to the long range transmissions that they have to perform to communicate with the aircraft. Thus the critical nodes and the cluster heads determine the lifetime of the network. Let $P_{0}$ and $P_{1}$ be respectively the average amounts of energy spent by a critical node and a cluster head during each cycle. Then to ensure a lifetime of $T$, we require:

$$
\frac{E_{1}}{P_{1}}=\frac{E_{0}}{P_{0}} \geq T
$$

where the equality of the first two terms ensures that both the critical nodes as well as the cluster heads expire at about the same time so that very little energy is wasted in the form of residual energy in the nodes when the network becomes unusable.

We assume that the nodes are asleep (with their radios turned off) in between the data gathering cycles. The nodes turn their radios on when the aircraft arrives for gathering updates. This results in a small duty cycle which in turn reduces the idle mode energy wastage. We assume that a data gathering cycle lasts for $\tau$ seconds. During these $\tau$ seconds all the nodes keep their radios in idle state. This is required because the nodes use multi-hop communication, and therefore cannot predict exactly when their neighbor nodes will send them a packet. Hence all the nodes have to keep their radios in idle state so as to be prepared to receive a packet. Since the radios are on during the entire data gathering phase, no extra energy is spent on receiving a packet. This assumption is justified by recent studies that have shown that the energy spent while receiving a packet is about the same as the energy spent in the idle mode [7]. If $l$ be the energy spent per second in the idle mode, then the total energy spent in the radio during each cycle is simply $l \tau$. We also ignore any packet collisions, by assuming that the cluster head nodes perform scheduling to ensure perfect synchronization. Packet collisions are a potential problem for the critical nodes since they have plenty of traffic to relay, and they are close to each other. We assume that the cluster head node is in-charge of this co-ordination. We also assume a simple propagation model in which the energy spent in the RF circuitry to transmit a packet over a distance $x$ is $\mu x^{k}$ where $k$ is the propagation exponent. Usually the value of $k$ is determined through on-site measurement, and varies between 2 and 4 depending on the surrounding environment. In environments with dense vegetation, the fall off is more drastic [8]. Since the aircraft is flying at high altitude (as compared to dimensions of the antenna on the nodes), we assume that the receiver antenna is in the far-field.

Since there are $n_{0}$ sensor nodes and $n_{1}$ cluster heads, there are $n_{0} / n_{1}$ sensor nodes in each cluster. There are exactly 4 critical nodes in each cluster which perform the function of relaying of packets from the $n_{0} / n_{1}-4$ sensor nodes (see Figure 2). Thus each critical node has to relay $\left(n_{0} / n_{1}-4\right) / 4$ packets. Besides this, the critical node also has to transmit its own packet. Let $E_{0}^{t}=\mu r^{k}$ be the amount of energy spent on transmission of a packet. Then the average energy 
spent by a critical node during each cycle is

$$
\begin{aligned}
P_{0} & =E_{0}^{t}\left(\frac{\left(\frac{n_{0}}{n_{1}}\right)-4}{4}\right)+E_{0}^{t}+l \tau \\
& =\frac{E_{0}^{t}}{4}\left(\frac{n_{0}}{n_{1}}\right)+l \tau \\
& =c_{0} \frac{n_{0}}{n_{1}}+c_{1}
\end{aligned}
$$

Each cluster head has to receive $n_{0} / n_{1}$ packets, aggregate them and transmit the aggregated packet to the aircraft. Let $E_{f}$ be the amount of energy spent in data computations during the aggregation of each packet by the cluster head, and $E_{1}^{t}=\mu H^{k}$ be the amount of energy required to transmit a packet from the cluster head to the aircraft. The cluster head also has to spend $l \tau$ amount of energy in the idle mode during each cycle. Therefore the average energy spent by a cluster head during each cycle is

$$
\begin{aligned}
P_{1} & =E_{f} \frac{n_{0}}{n_{1}}+E_{1}^{t}+l \tau \\
& =c_{2} \frac{n_{0}}{n_{1}}+c_{3}
\end{aligned}
$$

We have introduced the constants $c_{0}, c_{1}, c_{2}$ and $c_{3}$ for ease of notation.

Our objective is to minimize the cost of the network while ensuring connectivity and coverage of the area over $T$ cycles, i.e., given $r$ and $T$ we would like to determine $n_{0}, n_{1}, E_{0}$ and $E_{1}$ which minimize the cost of the network. Hence we formulate the optimization problem as follows:

$$
\begin{array}{cl}
\operatorname{minimize} & n_{0} \alpha_{0}+n_{1} \alpha_{1}+\beta\left(n_{0} E_{0}+n_{1} E_{1}\right) \\
\text { subject to } & n_{0}+n_{1} \geq \frac{1}{r^{2}} \\
& \frac{E_{1}}{P_{1}}=\frac{E_{0}}{P_{0}} \geq T
\end{array}
$$

Then the first constraint can be rewritten as:

$$
g_{1}(\bar{x})=\frac{1}{r^{2}}-n_{0}-n_{1} \leq 0
$$

Substituting for $P_{0}$ and $P_{1}$ in the equality constraint of (7) from (4) and (5) we obtain

$$
\begin{aligned}
& E_{1} P_{0}-E_{0} P_{1}=0 \\
& \Rightarrow h(\bar{x})=E_{1}\left(c_{0} \frac{n_{0}}{n_{1}}+c_{1}\right)-E_{0}\left(c_{2} \frac{n_{0}}{n_{1}}+c_{3}\right)=0
\end{aligned}
$$

Similarly from the inequality constraint of (7) we obtain

$$
E_{1} \geq T P_{1} \Rightarrow c_{3} T+c_{2} T \frac{n_{0}}{n_{1}}-E_{1}=g_{2}(\bar{x}) \leq 0
$$


Thus from (1), (8), (9) and (10) the optimization problem can be formulated as follows

$$
\begin{aligned}
\operatorname{minimize} & f(\bar{x}) \\
\text { subject to } & h(\bar{x})=0 \\
& g_{1}(\bar{x}) \leq 0 \\
& g_{2}(\bar{x}) \leq 0
\end{aligned}
$$

This is a standard Karush-Kuhn-Tucker (KKT) optimization problem [6]. We find the solution to this problem by solving:

$$
\begin{aligned}
\nabla f(\bar{x})+\lambda \nabla h(\bar{x})+\mu_{1} \nabla g_{1}(\bar{x})+\mu_{2} \nabla g_{2}(\bar{x}) & =\mathbf{0} \\
\text { with } \mu_{1} \geq 0, \quad \mu_{2} \geq 0, \quad \mu_{1} g_{1}(\bar{x})+\mu_{2} g_{2}(\bar{x}) & =0
\end{aligned}
$$

where $\lambda, \mu_{1}$ and $\mu_{2}$ are the constants of the KKT problem. From (1):

$$
\nabla f(\bar{x})=\left[\begin{array}{c}
\alpha_{0}+\beta E_{0} \\
\alpha_{1}+\beta E_{1} \\
\beta n_{0} \\
\beta n_{1}
\end{array}\right]
$$

From (9):

$$
\nabla h(\bar{x})=\left[\begin{array}{c}
\frac{E_{1} c_{0}-E_{0} c_{2}}{n_{1}} \\
-\frac{\left(E_{1} c_{0}-E_{0} c_{2}\right) n_{0}}{n_{1}^{2}} \\
-c_{3}-\frac{c_{2} n_{0}}{n_{1}} \\
c_{1}+\frac{c_{0} n_{0}}{n_{1}}
\end{array}\right]
$$

From (8):

$$
\nabla g_{1}(\bar{x})=\left[\begin{array}{c}
-1 \\
-1 \\
0 \\
0
\end{array}\right]
$$

From (10):

$$
\nabla g_{2}(\bar{x})=\left[\begin{array}{c}
\frac{c_{2} T}{n_{1}} \\
-\frac{c_{2} T n_{0}}{n_{1}{ }^{2}} \\
0 \\
-1
\end{array}\right]
$$


Substituting (13) to (16) in (11) and (12) we have the following four equations:

$$
\begin{aligned}
0= & \left(\alpha_{0}+\beta E_{0}\right)+\lambda\left(\frac{E_{1} c_{0}-E_{0} c_{2}}{n_{1}}\right)+\mu_{1}(-1) \\
& +\mu_{2}\left(\frac{c_{2} T}{n_{1}}\right) \\
0= & \left(\alpha_{1}+\beta E_{1}\right)+\lambda\left(\frac{\left(c_{2} E_{0}-c_{0} E_{1}\right) n_{0}}{n_{1}^{2}}\right) \\
& +\mu_{1}(-1)+\mu_{2}\left(-\frac{c_{2} T n_{0}}{n_{1}^{2}}\right) \\
0= & \left(\beta n_{0}\right)+\lambda\left(-c_{3}-\frac{c_{2} n_{0}}{n_{1}}\right)+\mu_{1}(0)+\mu_{2}(0) \\
0= & \left(\beta n_{1}\right)+\lambda\left(c_{1}+\frac{c_{0} n_{0}}{n_{1}}\right)+\mu_{1}(0)+\mu_{2}(-1) \\
0= & \mu_{1}\left(\frac{1}{r^{2}}-n_{0}-n_{1}\right)+\mu_{2}\left(c_{3} T+c_{2} T \frac{n_{0}}{n_{1}}-E_{1}\right)
\end{aligned}
$$

Assuming that a feasible solution exits, i.e., $n_{0}, n_{1}, E_{0}, E_{1}>0$, from (19), we get

$$
\lambda=\frac{\beta n_{0} n_{1}}{c_{3} n_{1}+c_{2} n_{0}}>0
$$

Hence from (20), we get

$$
\mu_{2}=\left(\beta n_{1}\right)+\lambda\left(c_{1}+\frac{c_{0} n_{0}}{n_{1}}\right)>0
$$

But from the KKT theorem, this implies that the corresponding inequality (10) becomes an equality, i.e.,

$$
c_{2} T+c_{3} T\left(\frac{n_{0}}{n_{1}}\right)-E_{1}=g_{2}(\bar{x})=0
$$

Using the value of $\lambda$ from (22) in (17),

$$
\begin{aligned}
& \mu_{1}=\left(\alpha_{0}+\beta E_{0}\right)+\lambda\left(\frac{E_{1} c_{0}-E_{0} c_{2}}{n_{1}}\right)+\mu_{2}\left(\frac{c_{2} T}{n_{1}}\right) \\
& =\alpha_{0}+\frac{\beta\left(c_{3} n_{1} E_{0}+c_{0} n_{1} E_{1}\right)}{c_{2} n_{1}+c_{3} n_{0}}+\mu_{2}\left(\frac{c_{2} T}{n_{1}}\right)>0
\end{aligned}
$$

since $\mu_{2}>0$. Hence

$$
\mu_{1}>0
$$

Again using the KKT theorem, this implies that the inequality of (8) becomes an equality, i.e.,

$$
\frac{1}{r^{2}}-n_{0}-n_{1}=g_{1}(\bar{x})=0
$$


So now we can re-formulate our problem as follows:

$$
\begin{aligned}
& \operatorname{minimize} f(\bar{x}) \\
& \text { subject to } h(\bar{x})=0 \\
& g_{1}(\bar{x})=0 \\
& g_{2}(\bar{x})=0
\end{aligned}
$$

Let $N$ denote the total number of nodes of both types. Then from (8) we obtain

$$
\begin{aligned}
n_{0}+n_{1} & =\frac{1}{r^{2}}=N \\
& \Rightarrow n_{0}=N-n_{1}
\end{aligned}
$$

From (10) and (9) we obtain the following expressions for $E_{0}$ and $E_{1}$

$$
\begin{aligned}
& E_{0}=T\left(c_{0} \frac{n_{0}}{n_{1}}+c_{1}\right) \\
& E_{1}=T\left(c_{2} \frac{n_{0}}{n_{1}}+c_{3}\right)
\end{aligned}
$$

Eliminating $n_{0}, E_{0}$ and $E_{1}$ from (1) using (27), (28) and (29), and after rearranging terms we obtain

$$
\begin{gathered}
f\left(n_{1}\right)=\alpha_{0} N+\beta T\left(c_{1}+c_{2}-2 N c_{0}\right)+n_{1}\left(\left(\alpha_{1}-\alpha_{0}\right)+\beta T\left(c_{3}+c_{0}-c_{1}-c_{2}\right)\right) \\
+\frac{\beta T c_{0} N^{2}}{n_{1}}
\end{gathered}
$$

To minimize the cost, we differentiate (30) with respect to $n_{1}$ and obtain the following solution for $n_{1}$

$$
n_{1}=\sqrt{\frac{c_{0} N^{2}}{\left(c_{3}+c_{0}-c_{1}-c_{2}\right)+\left(\frac{\alpha_{1}-\alpha_{0}}{\beta T}\right)}}
$$

Note that the second derivative of (30) is

$$
f^{\prime \prime}\left(n_{1}\right)=\frac{2 \beta T c_{0} N^{2}}{n_{1}{ }^{3}}>0 \quad \forall n_{1}>0
$$

Hence $f\left(n_{1}\right)$ is convex and therefore the local minimum is also the global minimum. Re-substituting for $c_{0}, c_{1}, c_{2}$ and $c_{3}$ from (4) and (5)

$$
c_{0}=\frac{\mu r^{k}}{4}, \quad c_{1}=l \tau, \quad c_{2}=E_{f}, \quad c_{3}=\mu H^{k}+l \tau
$$

Since for typical practical scenarios the aircraft altitude is much larger than the communication radius of each node, i.e., $H>r$, and usually the energy spent on communication $\left(\mu H^{k}\right)$ is much more as compared to the energy spent on 
computation $\left(E_{f}\right)$ for typical sensor nodes (see $\left.[5,7]\right)$, the $\mu H^{k}$ term dominates in $(31)$.

$$
\begin{aligned}
n_{1} & =\sqrt{\frac{\frac{\mu r^{k}}{4} N^{2}}{\mu H^{k}+\left(\frac{\alpha_{1}-\alpha_{0}}{\beta T}\right)}} \\
& =\frac{N^{1-\frac{k}{4}}}{\sqrt{4 H^{k}\left(1+\left(\frac{\alpha_{1}-\alpha_{0}}{\beta T \mu H^{k}}\right)\right)}}
\end{aligned}
$$

We used $N r^{2}=1$ from (26) to eliminate $r$. Thus we see that for a fixed $H$, the required number of cluster heads $n_{1}$ scales approximately as $N^{1-\frac{k}{4}}$.

In reality the sensor nodes used for surveillance have small sensing radii $(10 \mathrm{~m}$ to $100 \mathrm{~m})$, while the region to be monitored is usually large (a few square kilometers). Under such circumstances it is inevitable that a large number of nodes be used. To study such a scenario we consider the asymptotic behavior of $n_{1}$ as the number of nodes $N$ becomes large (or equivalently, as $r$ approaches zero). We note that for typical values of $k$ (between 2 and 4 ), the exponent of $N$ in (33) is always less than one. Hence $n_{1}$ scales slower than $N$. As a result $n_{0}=N-n_{1}$ scales approximately as $N$. This is something we would expect, since the majority of the $N$ nodes are used for sensing, while a few of them are used as cluster heads. Thus for large $N$, i.e., small $r$,

$$
\begin{aligned}
n_{0} & =N-n_{1} \approx N \\
& =\frac{1}{r^{2}}
\end{aligned}
$$

Thus we find that $n_{0} \approx N$ and $n_{1}$ scales approximately as $N^{1-\frac{k}{4}}$. Hence $n_{1}$ scales approximately as $n_{0}{ }^{1-\frac{k}{4}}$. Thus the propagation loss exponent $k$ determines the asymptotic behavior of the required number of cluster heads.

We can also obtain expressions for the battery energies of both types of nodes using (28) and (29) and re-substituting for $c_{0}, c_{1}, c_{2}$ and $c_{3}$ from (32).

$$
\begin{aligned}
& E_{0}=T\left(\frac{\mu \sqrt{H^{k}\left(1+\left(\frac{\alpha_{1}-\alpha_{0}}{\beta T \mu}\right)\right)}}{2 N^{\frac{k}{4}}}+l \tau\right) \\
& E_{1}=T\left(E_{f} N^{\frac{k}{4}} \sqrt{H^{k}\left(1+\left(\frac{\alpha_{1}-\alpha_{0}}{\beta T \mu H^{k}}\right)\right)}+\mu H^{k}+l \tau\right)
\end{aligned}
$$

In (34) we note the dependence of $E_{0}$ on $H$. This implies that higher the value of $H$, fewer the number of cluster head nodes (see (33)), and hence larger the cluster size. As the size of the cluster is higher, the critical nodes have more relaying to do, and consequently they need more battery energy. Thus $E_{0}$ increases as $H$ increases. 


\section{Case Study}

In our problem formulation, we have parameters $\alpha_{0}, \alpha_{1}$ and $\beta$ which correspond to the hardware cost of the two types of nodes and the proportionality constant for the battery cost. In general these parameters depend on the manufacturing process of the nodes. The cluster head nodes require powerful RF amplifiers, since they have to transmit data directly to the aircraft. Typical unmanned aerial vehicles (UAV) used for surveillance fly at altitudes of a few kilometers (see [9] for more details on UAVs). On the other hand, the sensor nodes have to communicate over distances of the order of 10 to $100 \mathrm{~m}$ (typical sensing radius). The cluster head nodes also require a more powerful CPU for data aggregation related computations and MAC and routing co-ordination. Hence we would expect $\alpha_{1}>\alpha_{0}$. However the exact dependence of $\alpha_{0}, \alpha_{1}$ and $\beta$ on these factors are difficult to account for without knowing the manufacturing process and the components involved.

Our approach provides designers with general guidelines to choose the system parameters. For example, consider the following scenario as a case study. Assume that a region of area $10 \mathrm{~km} \times 10 \mathrm{~km}$ is to be covered by a sensor grid. The nodes have a sensing radius $100 \mathrm{~m}$. A surveillance aircraft flying at an altitude of $10 \mathrm{~km}$ visits the region periodically. Since in our analysis we normalized the area of the region to unity, we must normalize all the distances in the case study by dividing them by $10 \mathrm{~km}$. Hence for this case study we have $r=0.01$ and $H=1$. The required number of cluster heads as a function of $\left(\alpha_{1}-\alpha_{0}\right) / \beta T \mu$ for $k=2$ and $k=3$ is plotted in Figure 3. The x-axis is a measure of the hardware cost difference between the two types of nodes divided by the battery parameter.

Depending on the manufacturing process, the value of $\left(\alpha_{1}-\alpha_{0}\right) / \beta T \mu$ can be determined. Using Figure 3 we can then determine the corresponding number of cluster head nodes required. The plots in Figure 3 have a step like shape because $n_{1}$, is rounded to the nearest integer. Note that the required number of sensor nodes is simply $1 / r^{2}=10^{4}$. We can also obtain the required battery energy of both types of nodes using (34) and (35).

\section{Conclusions and Future Work}

We studied the design of surveillance sensor grids from the point of view of lifetime of the network. In our study we considered a scenario in which two types of nodes are used; type 0 nodes, i.e., the sensor nodes and type 1 nodes, i.e., the cluster heads. Nodes are placed along grid points and a surveillance aircraft visits the area periodically to collect updates about the activity in the area. Our objective was to dimension the number of cluster heads, the number of sensor nodes and their battery energies so as to provide a network lifetime of at least $T$ cycles. During the lifetime of the network connectivity of the nodes as well as coverage of the area is ensured. We formulated and solved an optimization problem to minimize the overall network cost while attaining this objective. We found that the required number of cluster heads, $n_{1}$, scales approximately as 


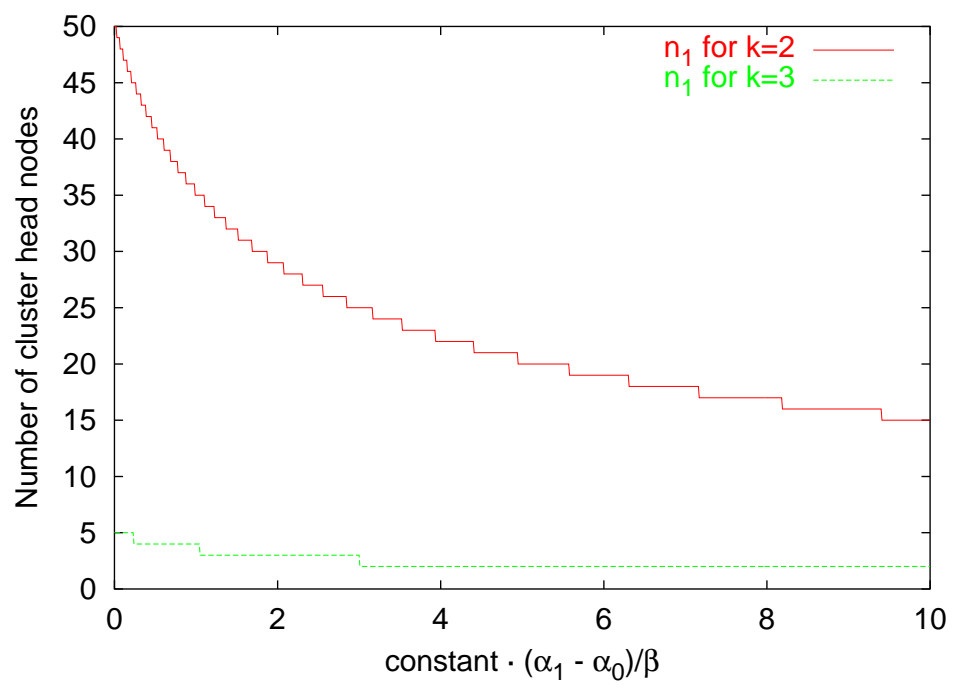

Fig. 3. Required number of cluster head as a function of hardware cost difference of two types of nodes.

$n_{0}{ }^{1-\frac{k}{4}}$ where $n_{0}$ is the number of sensor nodes, and $k$ is the propagation loss exponent.

The model of the sensor network that we consider in this work assumes a perfect grid deployment of nodes. However a more realistic scenario consists of random node deployment of (possibly) unreliable nodes. We would like to extend the results derived in this work to the random deployment scenario.

\section{References}

1. I.F. Akyildiz, W. Su, Y. Sankarsubramaniam and E. Cayirci, "Wireless Sensor Networks: a survey," Computer Networks, Vol. 38, pp. 393-422, March 2002.

2. M. Bharadwaj, T. Garnett and A.P. Chandrakasan, "Upper Bounds on Lifetime of Sensor Networks," IEEE International Conference on Communications (ICC'01), Helsinki Finland, June 2001.

3. C.F. Chiasserini, I. Chlamtac, P. Monti and A. Nucci, "Energy Efficient Design of Wireless Ad Hoc Networks," Proc. European Wireless, February 2002.

4. S. Bandyopadhyay and E. Coyle, "An Energy Efficient Hierarchical Clustering Algorithm for Wireless Sensor Networks," proc. IEEE INFOCOM'03, San Francisco, CA, April 2003.

5. W. Heinzelman, A. Chandrakasan and H. Balakrishnan. "An Application-Specific Protocol Architecture for Wireless Microsensor Networks," IEEE Transactions on Wireless Communications, Vol. 1, No. 4, October 2002.

6. E. Chong and S. Zak. An Introduction to Optimization, Second edition, Wiley and Sons, Inc., New York 2001.

7. G.J. Pottie and W.J. Kaiser, "Wireless Integrated Network Sensors," Communications ACM, 43 (5) (2000) 51-58. 
8. T. S. Rappaport, Wireless Communication, Prentice-Hall, 1996.

9. NASA GSFC/Wallops Flight Facility, Unmanned Aerial Vehicles. http://uav .wff . nasa.gov/index.html 